\title{
Transformations of Silver Nanoparticles in Wastewater Effluents: Links to Ag Bioavailability
}

\author{
Submitted to: \\ Environmental Science: Nano
}

\author{
AGIL AZIMZADA ${ }^{1}$, NATHALIE TUFENKJI ${ }^{1}$, and KEVIN J. \\ WILKINSON $^{2}$ \\ ${ }^{1}$ Department of Chemical Engineering, McGill University \\ Montreal, Quebec H3A 0C5, Canada \\ ${ }^{2}$ Department of Chemistry, University of Montreal \\ Montreal, Quebec H3C 3J7, Canada
}

* Corresponding Author. Phone: +1 (514) 343-6741; Fax: +1 (514) 343-7586; E-mail: kj.wilkinson@ umontreal.ca 


\section{ABSTRACT}

Wastewater effluents represent one of the main routes through which silver nanoparticles (Ag NPs) can reach natural aquatic environments, where they may interact with a range of organisms. However, in wastewaters, Ag NPs may undergo different chemical and physical transformations, which may alter NP toxicity towards aquatic organisms. The main objectives of this study are to characterize the dissolution and speciation of a Ag NP in a wastewater effluent and to assess its interactions with a model organism, Chlamydomonas reinhardtii (green alga), in the same medium. Experiments were conducted to distinguish the effect(s) of the wastewater matrix on the dissolution of Ag NPs and to determine whether the transformed NPs or the dissolved Ag species would be more bioavailable to $C$. reinhardtii. It was shown that in the wastewater effluent, $\mathrm{Ag}$ bioavailability was significantly reduced with respect to experiments using similar Ag concentrations in simple media. The substantial reduction in Ag bioavailability in wastewaters could be explained by the presence of high concentrations of organic/inorganic ligands, which complexed the Ag and the presence of substantial competing ions. Nonetheless, Ag NPs did appear to be bioavailable to C. reinhardtii at higher concentrations, as observed from bioaccumulation experiments comparing dissolved Ag and Ag NPs. Since the biouptake of Ag NPs could not be explained by concentrations of free $\mathrm{Ag}$ alone, it was hypothesized that complexed $\mathrm{Ag}$ species and/or small NPs may have contributed to biouptake. The results suggested that the chemistry of wastewaters had a significant effect on the bioavailability of Ag NPs, by modifying their physicochemical transformations. 
Keywords: silver, Chlamydomonas, toxicity, wastewater, nanoparticle, bioavailability.

\section{Introduction}

The extensive use of silver nanoparticles (Ag NPs) in consumer products and growing market demand has led to a significant increase in their production. Silver has a long historical record as an anti-bacterial agent [1-3], but the production of Ag NPs has led to the use of these materials in diverse market sectors, including textiles, cosmetics, food packaging and health supplements [4]. A major fraction of consumer products containing Ag NPs eventually find their way through waste disposal streams and into wastewater treatment plants [5-10]. Therefore, wastewater effluents represent a major route through which NPs can reach surface waters $[5,11]$, where aquatic organisms can be impacted.

Increasing emissions of Ag NPs have prompted discussions on the implications for the environment and human health [12-15]. A number of studies have been carried out to evaluate the bioavailability and toxicity of Ag NPs for various living organisms, but several challenges remain, including gaining a better understanding as to whether their impact is derived from the direct interaction of Ag NPs, the indirect release of Ag ions, or both [16]. For instance, Fabrega et al. [17] and Miao et al. [18] suggested that toxicity was directly attributed to the NPs, whereas Navarro et al. [19] and Piccapietra et al. [20] attributed the main toxicity of Ag NPs to the release of $\mathrm{Ag}^{+}$. Li et al. [21] observed that although Ag NP biouptake was mediated by the dissolved Ag, NPs were able to strongly sorb to the algal surface, which could impact their toxicity. Leclerc and Wilkinson [22] showed that bioaccumulation was largely due to the interfacial dissolution of Ag NP leading to the uptake of $\mathrm{Ag}$ ions, which were in turn reduced and precipitated intracellularly. Other 
authors have also concurred that $\mathrm{Ag}^{+}$and other dissolved $\mathrm{Ag}$ species could be generated locally at the biological interface from Ag NPs $[16,19]$.

There is a lack of consensus on the possible mechanisms through which Ag NPs become bioavailable and induce their toxic effects. The different conclusions may have arisen in part, due to differences in the type of Ag NPs, the organisms used in the different studies or the biological or toxicological endpoints that were tested. Furthermore, there is evidence that Ag NPs undergo near continual transformations in the environment through biotic or abiotic processes - the particular type and extent of the transformation typically being determined by the specific pathway and destination of the NP [5, 9, 10, 23, 24]. Any change in the physicochemical state of the NP will in turn likely impact its potential toxicity, as the bioavailability depends on chemical speciation (complexation and oxidation number), diffusive fluxes (related to NP size) and particle coating [25-27]. In a biochemically rich matrix of wastewater, Ag NPs may easily be transformed into numerous chemical species, including free $\mathrm{Ag}$ and $\mathrm{Ag}$ complexes, $\mathrm{Ag}_{2} \mathrm{O}, \mathrm{Ag}_{2} \mathrm{~S}$ and $\mathrm{AgCl}$ [23, 28-30]. For example, sulfidation is a prevalent process in wastewaters [9], which depends on $\mathrm{Ag}$ NP size and coating, the concentrations of reactive sulfide species $\left(\mathrm{H}_{2} \mathrm{~S}, \mathrm{HS}^{-}\right)$, and the oxygen content of the medium $[9,29]$. Furthermore, particle sulfidation may occur in a non-uniform fashion that leaves open the possibility of NP dissolution [29].

Numerous ligands in the wastewaters, notably thiols and dissolved organic matter and inorganic ligands such as chlorides, will modify the dissolution and speciation of $\mathrm{Ag}$ NPs [31-40]. Although dissolution has generally been shown to increase in the presence of these ligands, the dissolved Ag species will be mostly comprised of strong Ag complexes, which have typically been shown to be non-bioavailable [16, 20, 21, 31, 33, 41], with a 
few possible exceptions [42-44]. Indeed, studies on algae [16, 20-22], fish cells [33, 36, 45] and zebrafish embryos [35] have shown that Ag in ligand-rich media-particularly, those containing thiols (including amino acids and proteins), organic matter and chlorides - will be much less bioavailable than Ag in simpler media. Moreover, a few studies $[24,34,41,46,47]$ have suggested that in natural aquatic environments, dissolved Ag can be reduced back to small NPs, which are likely to interact differently with biological cells as compared to their original precursors.

Given the variety of environmental factors that can affect the transformations of $\mathrm{Ag}$ NPs in wastewaters, it is essential to rigorously characterize the different Ag forms, prior to their interaction with a biological cell. It is also important to assess the bioavailability of NPs in real, complex media (i.e., wastewater or surface water) as opposed to the simplified biological media in which the majority of toxicity experiments are performed. The combination of rigorous NP characterization and Ag speciation data with biological availability evaluations is necessary in order to gain more comprehensive insight into the risk of Ag NPs in complex environmental media.

Consequently, the objectives of this study were both to develop an in-depth understanding of Ag NP dissolution and speciation and to investigate the bioavailability of Ag NPs in an environmentally relevant medium of high importance, i.e., a wastewater effluent. Based upon the combined understanding gained from the characterization and bioavailability experiments, further insight into the nano-specific interactions of Ag NPs should be possible. 


\section{Materials and Methods}

Materials. All chemical reagents were purchased from Sigma-Aldrich (ACS reagent grade), except for $\mathrm{K}_{2} \mathrm{HPO}_{4}$ and $\mathrm{KH}_{2} \mathrm{PO}_{4}$ (ACS reagent grade; Fisher Chemical), $\mathrm{HCl}$ (trace metal grade; Fisher Chemical), $\mathrm{HNO}_{3}(67-70 \%$; $\mathrm{BDH}$ Aristar Ultra), $\mathrm{NaOH}$ (Acros Organics), tris-(hydroxymethyl)-aminomethane (BDH USP/EP grade, VWR) and $\mathrm{AgNO}_{3}$ (1000 mg Ag L ${ }^{-1}$; ICP-MS standard; Inorganic Ventures). Polypropylene tubes (15 and $50 \mathrm{~mL}$ ) from Fisher Scientific and polycarbonate shaker flasks $(1 \mathrm{~L})$ from VWR were used. Citrate/tannic acid coated Ag NPs $(50 \mathrm{~nm})$ were acquired from Nanocomposix (Econix silver) as an aqueous suspension with a nominal $\mathrm{Ag}$ concentration of $2.19 \mathrm{~g} \mathrm{~L}^{-1}$.

Wastewater effluent samples were collected from a municipal wastewater treatment plant in Montreal, Quebec, Canada. Milli-Q water (resistivity $>18.2 \mathrm{M} \Omega \mathrm{cm}$, organic

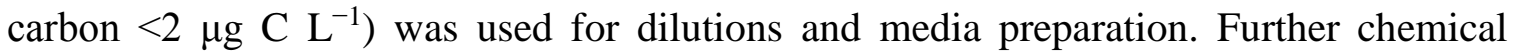
characterization of the effluent is provided in the Supplementary Information (Table S1). A simple biological medium, modified-TAP (M-TAP) (see detailed protocol in Table S2) (based on [48]) was used as a control medium in the dissolution and bioaccumulation experiments. In brief, M-TAP was prepared by diluting the salt composition (4x) of the standard tris-acetate-phosphate (TAP) algal growth medium and by substituting $\mathrm{Cl}^{-}$by $\mathrm{SO}_{4}{ }^{2-}$ and $\mathrm{NO}_{3}{ }^{-}$in order to minimize the potential for Ag precipitation.

Metal contamination was avoided by soaking flasks in $2 \% \mathrm{v} / \mathrm{v} \mathrm{HNO}_{3}$ for 24 hours, prior to rinsing them with Milli-Q water and drying them under laminar flow. To avoid biological contamination, materials and media used in the preparation of the algal growth media were autoclaved prior to use. Cysteine solutions were freshly prepared before use. 
Nanoparticle Characterization. Nanoparticle characterizations were performed in Milli-Q water (MQW), modified-TAP and wastewater effluent (WW) during short term (up to $75 \mathrm{~min}$ ) and/or long term (up to 7 day) exposures. For a few experiments designed to assess the effect of algal exudates on the dissolution of the Ag NPs, M-TAP solutions containing algae $\left(0.15 \mathrm{~cm}^{2} \mathrm{~mL}^{-1}, \sim 65,000\right.$ cells $\mathrm{mL}^{-1}, 1$ hour exposure) but no Ag, were filtered over $3.0 \mu \mathrm{m}$ nitrocellulose filter membranes (SSWP, Millipore). The filtrates, which were assumed to contain algal exudates but no algae, were then used as the NP exposure medium.

Ag NPs were characterized for their aggregation and dissolution using Inductively Coupled Plasma Mass Spectrometry (NexION 300X ICP-MS) in single particle mode (SPICP-MS). NP suspensions were diluted to between 100 and $400 \mathrm{ng} \mathrm{L}^{-1}$ in order to give a high ratio of signal (NP intensity) to background (dissolved Ag) that was appropriate for the dwell time that was employed $(500 \mu \mathrm{s})$. Data were typically acquired over $50 \mathrm{~s}$. Dilution levels and dwell times were optimized during preliminary experiments in order to avoid the concurrent ionization of more than one NP (leading to an overestimation of particle size and underestimation of particle number). Samples were nonetheless sufficiently concentrated so as to obtain statistically significant particle numbers. Transport efficiency was measured using a NIST (National Institute of Standards and Technology) reference (RM 8013) of $50 \mathrm{mg} \mathrm{L}^{-1}$ citrate stabilized gold NP with a nominal diameter of $60 \mathrm{~nm}$. The Syngistix nano application module integrated into the NexION software (version 1.1.4624) was used to acquire the time-resolved signals. To ensure the reliability of the measurements, blanks and quality control (QC) NPs (NIST 1640a) were run regularly (blanks after each sample and QC every 10 samples). 
As a complementary approach to estimate dissolution, ultracentrifugation experiments were performed using $25-200 \mu \mathrm{g} \mathrm{L}^{-1}$ of the Ag NPs in the wastewater effluent following 1 day of aging. In that case, $6 \mathrm{~mL}$ suspensions were centrifuged at 280,000 $\mathrm{g}$ for 15 min using a Sorvall mTX 150 Micro-Ultracentrifuge (ThermoFisher). Five mL of supernatant were carefully removed from each tube to avoid disturbing the pellets. Ag in NP form was calculated from the difference between the Ag measured in the supernatant (i.e., dissolved $\mathrm{Ag}$ ) and the total $\mathrm{Ag}$ in the initial samples. NP dissolution (\%) was determined by dividing Ag in the supernatant by the total Ag.

Dissolved Ag Speciation. A column with an ion exchange resin (IEC) (Chelex-100 resin, 50-100 mesh, wet capacity of $0.40 \mathrm{meq}^{-1}$, Sigma) was coupled to the ICP-MS $[25,49]$ in order to gain further insight into Ag speciation in the different media. The underlying assumption was that strong Ag complexes, anionic complexes and small NPs would pass through the resin column, whereas free Ag and labile Ag complexes would be retained by the resin (Table S3). This technique has previously been used with Ag NPs [50] and $\mathrm{ZnO}$ NPs [51], where it was shown that NP recovery through the IEC columns was very high — particle numbers and particle sizes measured with or without the IEC were not significantly different. Additionally, Hadioui et al. [50] (Fig. S1) showed that Ag NPs and strong Ag complexes would exit the resin immediately, leaving no detectable traces of Ag in the samples.

Identical aliquots of Ag NPs were tested for Ag dissolution using classical SP-ICPMS and SP-ICP-MS coupled to the IEC. The observed differences in total dissolved Ag measured with and without the IEC was attributed to free or labile Ag, whereas Ag that passed through the IEC was considered to be either small NPs (below the size detection 
limit) or strong Ag complexes (Table S3). It is of note that the instrumental detection limit for the ICP-MS was $0.02 \mathrm{ng} \mathrm{L}^{-1}$ (under the applied conditions) and that Ag levels in the original WW sample were below the detection limit. In the M-TAP (control) medium, where the exact composition was known, Visual MINTEQ software (version 3.0) was used for the determination of Ag speciation.

Algal Cultures. In this study, Chlamydomonas reinhardtii (wild type C125 from the Chlamydomonas resource center) was used as a model organism. Algal culturing [22, 48] involved two transfer steps. First, $75 \mathrm{~mL}$ of a defined algal growth medium (4× diluted tris-acetate-phosphate medium (TAP)) was inoculated with $C$. reinhardtii cells from a Petri dish (agar with TAP). The inoculated algal suspension was incubated at $20{ }^{\circ} \mathrm{C}$ under $24 \mathrm{~h}$ lighting with $100 \mathrm{rpm}$ of rotary agitation, until the algae reached their exponential growth phase [22]. An aliquot of the algal culture was then transferred to a fresh $400 \mathrm{~mL}$ of algal growth medium ( $4 \times$ diluted TAP) where it was incubated until a new exponential growth phase was attained. Cell numbers were measured using a Multisizer 3 particle counter (Beckman Coulter). Measured cell densities (number or surface area) were used to determine the growth phase as well as to ensure constant algal cell densities in the experimental media. Two hundred $\mathrm{mL}$ of an algal suspension was centrifuged at $3,000 \mathrm{~g}$ for 3 min in order to separate the algal cells. The pellet was washed with M-TAP solution and the supernatant was discarded. This washing procedure was repeated twice $(2$ min of centrifugation) and the algal concentrate was diluted in $10 \mathrm{~mL}$ of the M-TAP.

Bioavailability Experiments. Ag NPs (or $\mathrm{AgNO}_{3}$ ) were pre-equilibrated in the medium of interest-M-TAP or the wastewater effluent - for 24 hours prior to the exposure of the algae. Exposure media were covered with aluminum foil to protect them from light 
and placed on a rotary shaker at $100 \mathrm{rpm}$. Bioaccumulation experiments (technical triplicates) were begun by adding a small number of algal cells from the concentrated algal stock suspension into the exposure medium (containing Ag) in order to obtain a final cell density of $0.15 \mathrm{~cm}^{2} \mathrm{~mL}^{-1}\left(\sim 65,000\right.$ cells $\left.\mathrm{mL}^{-1}\right)$ [22]. In a previous study, this cell density was shown to be low enough to ensure that Ag concentrations did not decrease significantly over the duration of the short term experiments [22]. Given its ability to strongly bind $\mathrm{Ag}^{+}$ and limit its bioavailability $[19,22,52]$, cysteine was added to stop (or at least significantly reduce) bioaccumulation at selected time-points with the expectation that it would also remove Ag bound to the outside of the cell (i.e., sorbed Ag or loosely-bound Ag particles). Although the Ag fraction that is not extracted by the cysteine wash has been referred to as algal $\mathrm{Ag}$ or $\mathrm{Ag}$ biouptake below, it is an operationally defined fraction that is based upon two major assumptions: (i) the cysteine wash will remove all Ag bound to the outside of the cell [22] and (ii) adsorption is a rapid process as compared to internalization [53]. This fraction may nonetheless also include Ag NPs that are irreversibly bound to the cell surface.

Although filter-based approaches have been used previously [20, 22, 54], the testing of filter membranes for potential adsorptive losses requires additional care in order to ensure acceptable recoveries in the mass balances. Adsorptive losses can be a source of concern, particularly in the experiments conducted with NPs, as they may be more easily retained by the membranes than the dissolved ions. Indeed, for preliminary biouptake experiments with NPs, significant adsorptive losses were observed (up to 80\%) when nitrocellulose membranes were used (Fig. S2). Thus, the experimental approach for Ag NPs was modified slightly from that for $\mathrm{AgNO}_{3}$ in order to minimize adsorptive losses to less than $5 \%$ and to achieve acceptable mass recoveries. Triplicate, $45 \mathrm{~mL}$ samples were 
mixed with $5 \mathrm{~mL}$ of $5 \times 10^{-2} \mathrm{M}$ cysteine, gently shaken for $1 \mathrm{~min}$ (pre-filtration cysteine wash), and then filtered over $3.0 \mu \mathrm{m}$ nitrocellulose membranes (SSWP, Millipore) (for $\mathrm{AgNO}_{3}$ experiments) or over $3.0 \mu \mathrm{m}$ polycarbonate filter membranes (TSTP, Isopore) (for $\mathrm{Ag}$ NP experiments). Filtered algal cells (diameter of $\sim 6 \mu \mathrm{m}$ ) were washed three times with $5 \mathrm{~mL}$ of $5 \times 10^{-3} \mathrm{M}$ cysteine [53]) (post-filtration cysteine wash). In the case of Ag NPs, independent control runs (before adding algae) were conducted to verify adsorptive losses to the filter membranes (polycarbonate), whereas in the case of $\mathrm{AgNO}_{3}$, two superimposed filter membranes (nitrocellulose) [22] were employed, where the bottom membrane acted as the adsorption control. All control experiments for the adsorptive losses included the pre- and post-filtration cysteine washing steps, to ensure that any potential adsorptive losses due to the formation of Ag-cysteine oligomers [41, 55] were well accounted for.

Filter membranes were digested with $300 \mu \mathrm{L}$ of $67-70 \% \mathrm{HNO}_{3}$ (Aristar Ultra) for 8 hours at $85^{\circ} \mathrm{C}$ using a DigiPREP digestion system (SCP Science). Samples were diluted to $3 \% \mathrm{v} / \mathrm{v} \mathrm{HNO}_{3}$, one day before the ICP-MS measurements. Similarly, samples from the filtrates and the exposure media, before and after algal addition, at each time-point, were acidified and kept at $85{ }^{\circ} \mathrm{C}$ for 8 hours, before being diluted to $3 \% \mathrm{v} / \mathrm{v} \mathrm{HNO}_{3}$. Mass balances were performed and any data points outside the $85-115 \%$ range were discarded.

\section{Results and Discussion}

Nanoparticle sizes. For $400 \mathrm{ng} \mathrm{L}^{-1}$ of Ag NPs in a wastewater effluent, mean diameters of $51.6 \pm 0.1 \mathrm{~nm}, 48.6 \pm 0.1 \mathrm{~nm}, 51.8 \pm 0.4 \mathrm{~nm}$ and $51.0 \pm 0.3 \mathrm{~nm}$ were determined after 1, 3, 5 and 7 days, respectively. These SP-ICP-MS results showed that the 
particle diameters did not change significantly over time and were in good agreement with the original particle size of $54.7 \pm 6.6 \mathrm{~nm}$, provided by the manufacturer (determined by transmission electron microscopy). Additional measurements obtained in the wastewater effluent, in the M-TAP medium and in the Milli-Q water for Ag NP concentrations of 100 and $400 \mathrm{ng} \mathrm{L}^{-1}$ are provided in Table S4. For measurements performed over 75 mins in the M-TAP medium (400 $\mathrm{n} \mathrm{L}^{-1} \mathrm{Ag} \mathrm{NP}$ ), without and with algal exudates, the NPs had stable diameters of $53.6 \pm 0.3 \mathrm{~nm}$ and $51.9 \pm 1.0 \mathrm{~nm}$, respectively.

Nanoparticle dissolution. Ag NP dissolution was determined by SP-ICP-MS in the wastewater, M-TAP and Milli-Q water (Fig. 1). Total Ag concentrations in the experimental tubes decreased slightly (0-4 \% for $10 \mu \mathrm{g} \mathrm{L}^{-1}$ of Ag; up to $30 \%$ for $100 \mathrm{ng}$ $\mathrm{L}^{-1}$ of Ag; Fig. S3), likely due to adsorptive losses. When measured concentrations of dissolved $\mathrm{Ag}$ were normalized by the measured concentrations of total $\mathrm{Ag}$, it was clear that particle dissolution increased with time (Fig. 2). Indeed, for $100 \mathrm{ng} \mathrm{L}^{-1}$ of the Ag NP in wastewater, dissolution increased dramatically, with the proportion of dissolved $\mathrm{Ag}$ (includes free and complexed Ag) reaching 63\% after 7 days. For the same concentration of Ag NPs in Milli-Q water, the particles were much more stable with only $4.7 \pm 2.9 \%$ of dissolved Ag after 7 days, whereas in the algal growth media (M-TAP), the dissolution attained 27\%, where it was stable for at least 3 days.

\section{[Figure 1]}

The effect of algal exudates on the dissolution of the Ag NPs is shown in Figure 2. For experiments occurring over several days, dissolution (\%) nearly doubled in the M-TAP medium containing algal exudates as compared to the standard M-TAP (Fig. 2A). Similar 
measurements were conducted to determine Ag NP dissolution at shorter time scales (within 75 min.); the results showed that the dissolution was faster and more important than for the control medium containing no exudates. The effect of the algal exudates on Ag NP dissolution was rapid (Fig. 2B) and not observed in the medium that did not contain the exudates (over 75 mins).

\section{[Figure 2]}

Validation of SP-ICP-MS results. When analyzing SP-ICP-MS results, it is necessary to acknowledge that the quantification of dissolved and NP Ag can be influenced by the lower size cut-off (or size detection limit) of the instrument. Nanoparticles that are smaller than this threshold value are effectively considered to be dissolved. Since the data processing for SP-ICP-MS is based on accurately distinguishing the signals of the nanoparticles from those of the dissolved metal (i.e., free and complexed Ag) $[50,56]$, the cut-off becomes an important parameter-especially when comparing Ag NP dissolution in media with different chemical compositions. Furthermore, it is critical to ensure that the often observed increase in the size detection limit in complex media (due to the increase in dissolved metal) does not result in artefactual increase in particle size (due to the greater attribution of small particles to the dissolved fraction) [49]. Using our SP-ICP-MS setup, the lower size cut-off of the instrument was approximately $17 \mathrm{~nm}$, whereas the smallest detected particles had diameters of $20.9 \pm 0.1 \mathrm{~nm}$ and $21.9 \pm 1.7 \mathrm{~nm}$ (Table S5) in the MTAP and the wastewater effluent, respectively. Although the cut-off values did increase in complex media (indicating greater dissolution), increases were relatively small and not sufficient to explain the increased dissolution of the NPs in the WW or M-TAP media as compared to the Milli-Q water (Fig. 1). Although it is impossible to completely neglect the 
masking of small NPs [49], most of the observed dissolution could be attributed to the interaction of medium components with the NPs. Combined with increased values of dissolved Ag, the decrease in particle numbers (Fig. S4) effectively show that in the wastewater effluent, a large fraction of the original NPs was either transformed into i) dissolved (free or complexed Ag) or ii) smaller NP (i.e., below size detection limit).

The results from complementary ultracentrifugation experiments (Table 1) were used to analyze the implications of SP-ICP-MS data. Following $15 \mathrm{~min}$. of ultracentrifugation at $280,000 \mathrm{~g}, \mathrm{Ag}$ concentrations in the supernatant corresponded to only $1.7 \pm 0.3 \%$ of the initial concentration of $\mathrm{Ag}$, in contrast to the $27.2 \pm 5.6 \%$ that was determined by SP-ICP-MS. Two explanations are likely to explain this apparent inconsistency: (i) the techniques function at very different concentration levels (ppb vs. ppt); (ii) in ultracentrifugation, adsorptive losses were likely greater for the dissolved Ag as compared to Ag NP. Indeed, the high dilution requirement for the samples analysed by SP-ICP-MS is likely to contribute to the higher \% dissolution observed by this technique since Ag NP dissolution is known to increase with decreasing particle concentration [49, 57]. Furthermore, although very little Ag was detected in the supernatant following ultracentrifugation, measured initial concentrations were significantly lower $(\sim 40-50 \%)$ than nominal concentrations. It is reasonable to assume that adsorptive losses of the dissolved (positively charged) $\mathrm{Ag}^{+}$to the container walls occurred to a greater extent than that of the highly stabilized (negatively charged) NPs [58], in which case, ultracentrifugation would underestimate dissolution. Based upon the above considerations, SP-ICP-MS is likely to provide an upper limit for the dissolution, whereas ultracentrifugation would be more likely to give a lower limit. 


\section{[Table 1]}

Wastewater medium and algal exudates enhance dissolution. The combined results of the SP-ICP-MS and the ultracentrifugation strongly indicated that NP dissolution and their transformation to smaller NPs increased: (i) with time; (ii) with increased dilution; and (iii) in complex media, i.e., in wastewater and in the M-TAP with algal exudates. Liu et al. [57] showed that $\mathrm{Ag}^{0}$ is not a stable (equilibrium) species when in the presence of significant dissolved $\mathrm{O}_{2}$ and at environmentally relevant $\mathrm{pH}$ (the wastewater effluent had a $\mathrm{pH}$ of $\sim 7.7-7.8)$. In this case, especially for low NP concentrations, oxidation and dissolution are thermodynamically favourable. Furthermore, increased Ag NP dissolution in the biochemically rich matrices is consistent with the complexation of free $\mathrm{Ag}$ in solution $[19,41,52,59,60]$, which will drive both the oxidation of the Ag NPs and the desorption of surface bound $\mathrm{Ag}^{+}$[49]. Observations of increased dissolved $\mathrm{Ag}$ are entirely consistent with both chemical thermodynamics $[61,62]$ and with numerous studies $[31,32,34,37$, $40,41]$ where the ligand effects on the dissolution of Ag NPs have been demonstrated. Among these ligands, thiol-compounds, such as cysteine, gluthathione and mercaptoethanol, are particularly relevant as they can form very stable complexes with $\mathrm{Ag}^{+}$ [31]. Indeed, Sigg and Lindauer [34] and Gondikas et al. [41] have shown that the dissolution rate of Ag NPs was enhanced with increasing concentrations of cysteine. Other organic ligands, such as the fulvic acids may also increase dissolution of the Ag NPs - for example, Collin et al. [37] showed that the dissolution of partly-sulfidized Ag NPs increased in the presence of the Pony lake fulvic acid. While chlorides in wastewaters can precipitate $\mathrm{Ag}^{+}$as $\mathrm{AgCl}_{(\mathrm{s})}$, at sufficiently high concentrations, they are also expected to form soluble chlorosilver complexes (i.e., $\mathrm{AgCl}_{2}{ }^{-}, \mathrm{AgCl}_{3}{ }^{2-}, \mathrm{AgCl}_{4}{ }^{3-}$ ) that could further 
favour the dissolution of Ag NPs [33, 40]. Wastewaters are indeed rich with such entities [24, 63, 64], including organic (e.g., fulvic and humic acids, amino acids, proteins) or inorganic (e.g., chlorides) ligands that will both influence Ag NP dissolution and control the speciation of the dissolved $\operatorname{Ag}[31,59]$. Finally, dissolution can also be affected by the ionic strength of the medium, which is high in the wastewaters due to the presence of numerous salts [65]. Chambers et al. [40] provided evidence that the release of dissolved Ag from Ag NPs increased under increased ionic strength conditions.

Microorganism-produced compounds may also play a critical role in the dissolution of Ag NPs. For example, Wu et al. [38] showed that dissolution of Ag NPs increased substantially when in the presence of a number of organisms. Specifically, algae have been shown to excrete $\mathrm{H}_{2} \mathrm{O}_{2}$ [66], which can oxidize the surface of the Ag NP, leading to $\mathrm{Ag}^{+}$ release. In addition, algal exudates contain numerous organic compounds, including thiols, proteins or other exopolymeric substances (EPS) that are a potential source of ligands for $\mathrm{Ag}^{+}[67]$ and for which the concentrations may increase in response to metal exposure [39, $68]$.

Characterization of dissolved Ag. As the bioavailability of trace metals is most often predicted by the concentration of free ion, irrespective of the metal complexes [43, 69], the characterization of dissolved Ag speciation in the medium becomes very valuable. Based on the results of IEC-SP-ICP-MS, in the simplified growth medium (M-TAP), approximately $75 \%$ of dissolved $\mathrm{Ag}$ species could be attributed to the free or weakly-bound $\mathrm{Ag}$ fraction, whereas in the wastewater or in the M-TAP amended with algal exudates, over $82 \%$ of the $\mathrm{Ag}$ did not react with the ion exchange column (i.e. Ag was found mainly as either strong Ag complexes and/or small NPs) (Fig. 3 and Table S3). Indeed, the results 
are a confirmation that there was more dissolution in the complex waters, but that dissolved $\mathrm{Ag}$ in these media was largely complexed. Given that free Ag and labile Ag complexes are far more likely to be bioavailable $[16,19,43,69]$ - and if the only mechanism of bioavailability for nanoparticulate $\mathrm{Ag}$ is dissolution - it is possible to predict that the $\mathrm{Ag}$ will be far less bioavailable in the wastewater and that bioavailable Ag will decrease with time.

\section{[Figure 3]}

Ag bioavailability in the wastewater. Finally, metal uptake by the green alga $C$. reinhardtii was used as a basis to estimate the bioavailability of the different forms of $\mathrm{Ag}$. Algae were first exposed to $0.2-1.0 \mu \mathrm{mol} \mathrm{L}^{-1}$ of $\mathrm{AgNO}_{3}$ in the M-TAP medium over 40 min. Biouptake increased linearly with time (Fig. S5), consistent with the Biotic Ligand Model and previous literature results [22, 44]. When algae were exposed to similar concentrations (0.2-1.3 $\mu \mathrm{mol} \mathrm{L}-1)$ of $\mathrm{AgNO}_{3}$ in the wastewater effluent (Fig. 4B), much lower Ag internalization fluxes were observed, with fluxes that were not significant at 0.2

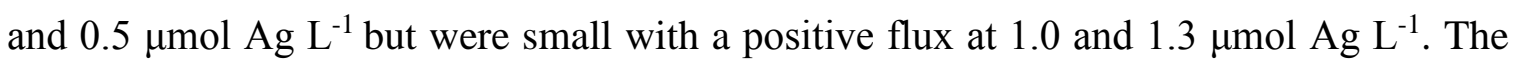
results clearly demonstrated that the bioavailability of $\mathrm{Ag}$ was largely or completely reduced when algae were exposed to $\mathrm{AgNO}_{3}$ in a wastewater medium. This observation was intuitively expected due to the presence of various chemical components in the wastewater that could lead to reduced bioavailability including i) increased complexation $[16,20-22,31,33,36]$ of $\mathrm{Ag}^{+}$by organic/inorganic ligands that render them nonbioavailable and ii) increased presence of ions in the wastewater that compete for $\mathrm{Ag}$ biouptake (competition) [42, 48, 70-73]. In contrast with the majority of previous studies where bioaccumulation experiments were conducted in simple growth media [19, 20, 22], 
Ag bioavailability in the wastewater was greatly reduced due to the complex nature of the medium.

\section{[Figure 4]}

Due to the low exposure concentrations, biouptake fluxes for the algae exposed to Ag NPs were more challenging to quantify. The use of polycarbonate filters for the Ag NP suspensions did minimize sorptive losses to less than $5 \%$ (for $[\mathrm{Ag}]$ as low as $0.2 \mu \mathrm{mol} \mathrm{L^{-1 }}$ ) (Fig. S4). At the lowest exposure concentrations of Ag NPs $\left(0.5\right.$ and $\left.0.8 \mu \mathrm{mol} \mathrm{L} \mathrm{L}^{-1}\right)$, biouptake fluxes were not significantly different from 0 (one-tailed t-test; $\mathrm{p}<0.05$ ) (Table S6). However, when biouptake experiments were performed using $1.3 \mu \mathrm{mol} \mathrm{L}-1$ (or 2.0 $\mu$ mol $\mathrm{L}^{-1}$, see Table S6) of Ag NPs, uptake fluxes were significantly greater than those obtained following the addition of equivalent or lower concentrations of $\mathrm{AgNO}_{3}$ (Fig. $5 A \& B)$. These results indicated that, in the wastewater, the Ag from the NPs appeared to be bioavailable, potentially resulting from their dissolution and production of small NPs or Ag complexes, as indicated by the SP-ICP-MS and ultracentrifugation experiments. Since the IET-ICP-MS experiments showed that most of the dissolved Ag resulting from the Ag NPs was in the form of strong complexes, biouptake in the wastewater could not be predicted from free $\mathrm{Ag}$ alone, as was shown previously for this alga in simplified media [22]. Indeed, if SP-ICP-MS (Fig. 1) and IET-ICP-MS (Fig. 3C) experiments performed with Ag NPs were used as a basis to determine free Ag, concentrations would be below $0.08 \mu \mathrm{mol} \mathrm{L}^{-1}$ for $1.3 \mu \mathrm{mol} \mathrm{L}-1$ of the $\mathrm{Ag} \mathrm{NP}$ suspension. These are $\mathrm{Ag}^{+}$concentrations that are below the level of free Ag shown to cause significant biouptake (with the protocol and instruments used in this study) (Fig. 4A). Therefore, the results strongly suggest that the 
production of smaller NPs or Ag complexes (resulting from transformations of the Ag NP in the wastewater) contributed to the overall biouptake.

\section{[Figure 5]}

Based upon our results, it is impossible to discount the potential contribution of small Ag NPs to the biouptake. Although the cysteine wash is thought to remove cell wall bound Ag NPs, the observed non-zero intercept suggests that some NPs may simply have been adsorbed on the cell surface. These small adsorbed NPs may have subsequently been internalized or have contributed to biouptake following their oxidative dissolution [19, 22]. A similar suggestion was made in the study of Ag NP interactions with a different alga, Euglena gracilis [21]. Such a result could be facilitated by the relatively high ionic strength of the wastewater that would compress the electrical double layer and facilitate the sorption of small Ag NPs by the cell [40]. In that case, the (interfacial) dissolution of the surfacebound NPs would be expected to contribute to the bioavailability and explain the unexpectedly high biouptake fluxes observed for the Ag NPs [16].

In summary, it is important to note that Ag bioavailability did decrease substantially due to complexation (and/or competition) in the wastewater effluent in comparison to simple media (fluxes in M-TAP were greater than those determined in the wastewaters (Fig. 4B\&5B)). Furthermore, little bioavailability could be directly attributed to either the ionic or particulate forms of Ag. We postulate that the Ag complexes and NPs function effectively as a Ag buffer with the capacity to locally buffer concentrations of free Ag. Nevertheless, as the results pointed out, the occurrence of biological internalization and/or 
strong surface adsorption of small Ag NPs can be facilitated by the physicochemical transformations of the NPs in the complex wastewater medium.

Environmental implications and challenges. In a wastewater effluent, Ag NPs appeared to be more bioavailable than could be predicted based solely upon the concentrations of free Ag. This observation is in stark contrast with previous studies [20, 22] that have been performed using simple biological media, where NP biouptake was largely attributed to dissolved Ag (presumably ionic Ag). Nonetheless, such a result is still consistent with previous studies $[16,19,20]$ that have suggested that Ag NP bioavailability to algal cells could be mediated by the release of $\mathrm{Ag}^{+}$at the surface of the organism (interfacial dissolution). Another implication of the study is that the biological internalization and adsorption of NPs can increase in a wastewater medium, despite the reduction in the bioavailability of dissolved Ag species, which was attributed to strong complexation and competition effects. Indeed, the potential increase in the concentration of small NPs over time in a wastewater medium - through the dissolution or disaggregation of large NPs or the reduction of dissolved Ag-may have been a potential cause of NP bioavailability that was higher than predicted based on free Ag concentrations. However, the analytical difficulties in distinguishing Ag complexes from very small Ag NP (i.e., those below the detection limits of the SP-ICP-MS) limits our capacity to draw more robust conclusions on the precise form of bioavailable Ag. Nonetheless, it is clear that the bioavailability of Ag NP in wastewaters will be largely determined by the interactions with physiochemical elements that are present, however, neither free $\mathrm{Ag}$ nor total $\mathrm{Ag}$ concentrations will be a sufficient predictor of biological effects. 
Despite the progress achieved in this study with respect to the reliability of the methodology and environmental relevance, several challenges still remain. One challenge is to develop a technique to detect, quantify and discriminate cell-internalized Ag (NPs or dissolved $\mathrm{Ag}$ ) at the lowest ranges of environmentally relevant concentrations, as the biouptake quantification with the current methodology is limited to $\sim 10 \mu \mathrm{g} \mathrm{L}^{-1}$. NPs are subjected to different environmental factors that will affect their persistence in wastewaters. In a laboratory setting, simulation of real environmental conditions is a challenging task. Extraction of NP samples from an environmental matrix with minimal alterations to their physicochemical properties would be most useful for exposure experiments. Clearly, the numerous physicochemical transformations occurring in the complex wastewaters will greatly affect the bioavailability of the nanomaterials.

\section{Acknowledgements}

This research was supported by the Natural Sciences and Engineering Research Council of Canada (NSERC), Environment Canada, Perkin Elmer, and the NSERC CREATE award program. Special thanks go to J.-A. Rowell (University of Montreal) for her contribution to the biouptake experiments, M. Hadioui (University of Montreal) for assistance with the SP-ICP-MS and M. Mitzel (McGill University) for his collaborative work with ultracentrifugation experiments. 


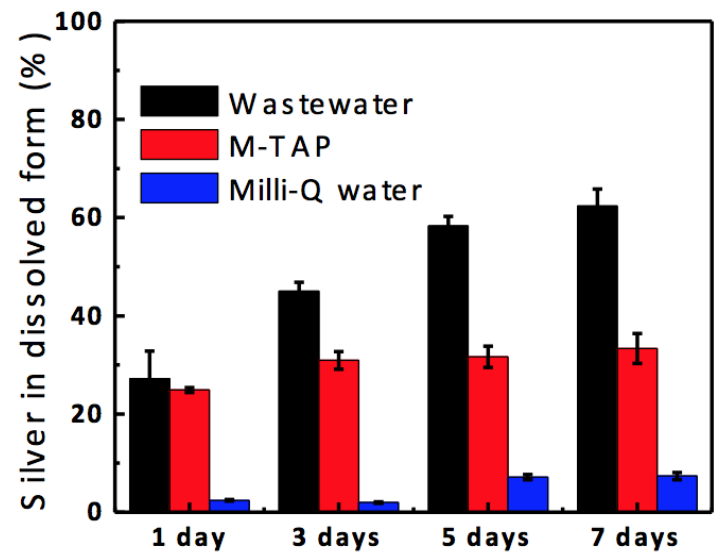

Figure 1. Dissolution of Ag NPs (initially $100 \mathrm{ng} \mathrm{Ag} \mathrm{L}^{-1}$ ) in wastewater effluent, modifiedTAP medium (M-TAP) and Milli-Q water after 1, 3, 5 and 7 days as quantified by SP-ICPMS. Dissolved Ag is expressed as a proportion of the total measured Ag (mean \pm standard deviation, $n=3$ ).
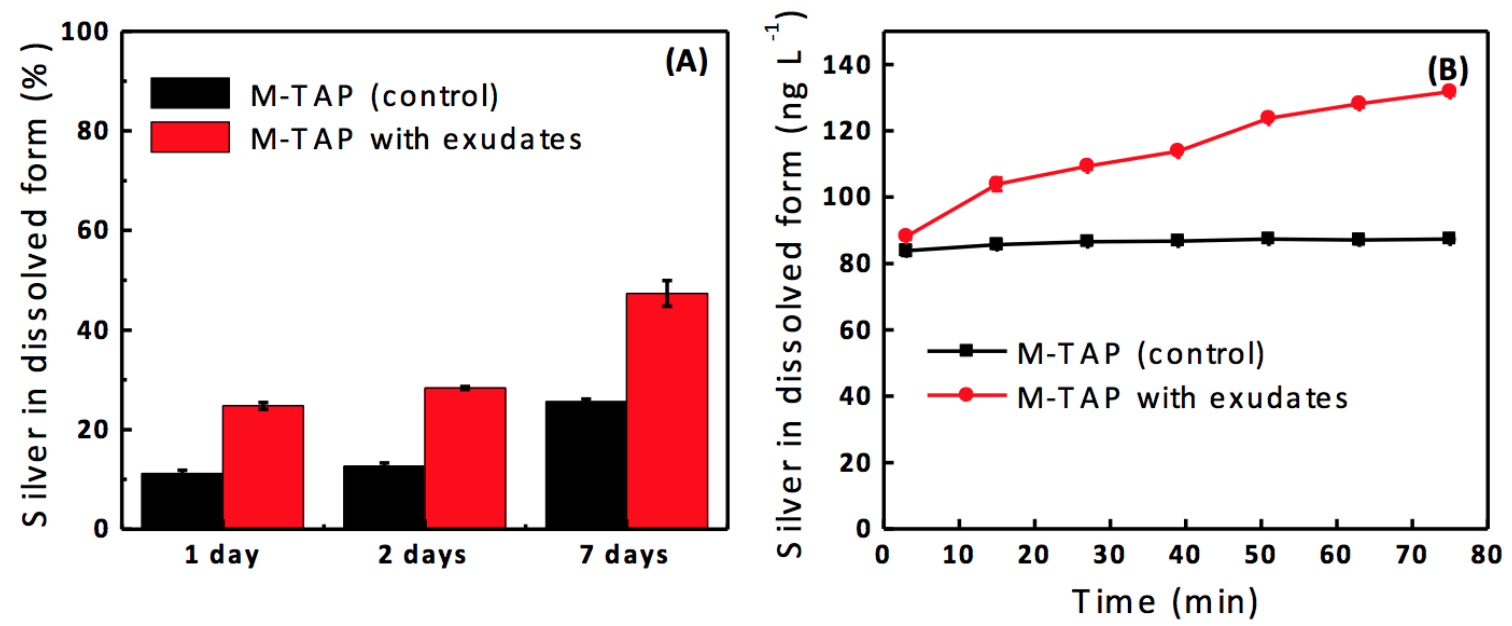

Figure 2. Dissolution of Ag NPs (nominally $400 \mathrm{ng} \mathrm{L}^{-1}$ ) in the modified-TAP in the presence and absence of algal exudates. A) Proportion of $\mathrm{Ag}(\%)$ in dissolved form (mean \pm standard deviation, $\mathrm{n}=3$ ) after 1, 2, or 7 days and B) Short term dissolution measurements (mean \pm standard deviation, $\mathrm{n}=3$ ) performed over 75 mins and measured in the M-TAP medium, with or without algal exudates. Determinations of dissolved Ag were made by SP-ICP-MS. 

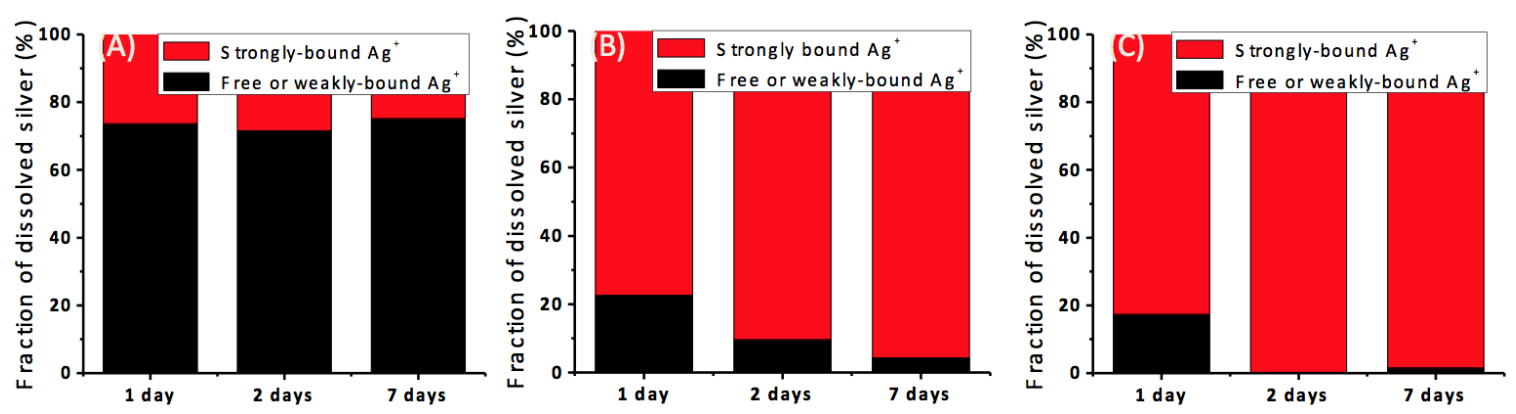

Figure 3. Ag speciation determinations for dissolved Ag species (from Ag NPs) based on IET-SP-ICP-MS in A) modified-TAP growth medium B) modified-TAP in the presence of algal exudates and $\mathrm{C}$ ) wastewater effluent.
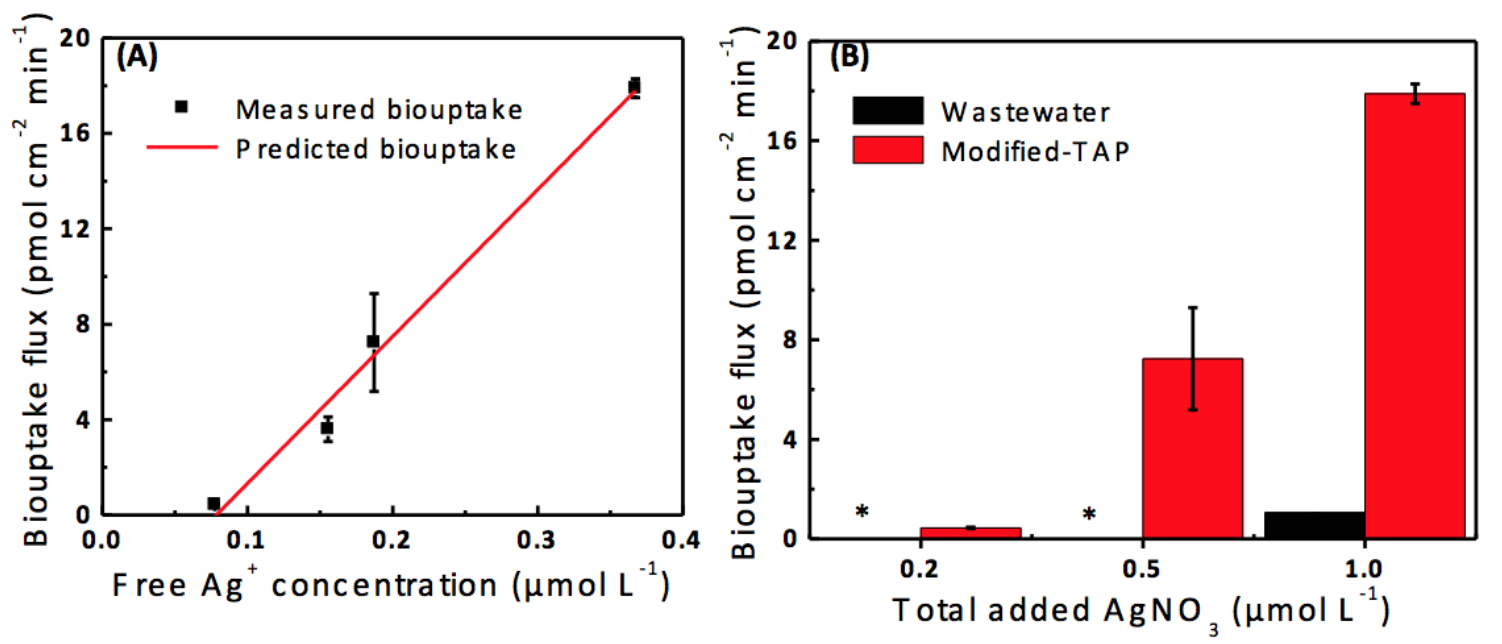

Figure 4. Determined biouptake fluxes (mean \pm standard error, $\mathrm{n}=3$ ) for algal cells $(C$. reinhardtii) exposed to $\mathrm{AgNO}_{3} \mathrm{~A}$ ) as a function of free $\mathrm{Ag}^{+}$concentrations (in modifiedTAP predicted using Visual MINTEQ) and B) in modified-TAP and wastewater effluent media as a function of total added Ag. (*) refers to fluxes determined in the wastewater that were not significantly different $(\mathrm{p}<0.05)$ from zero. 

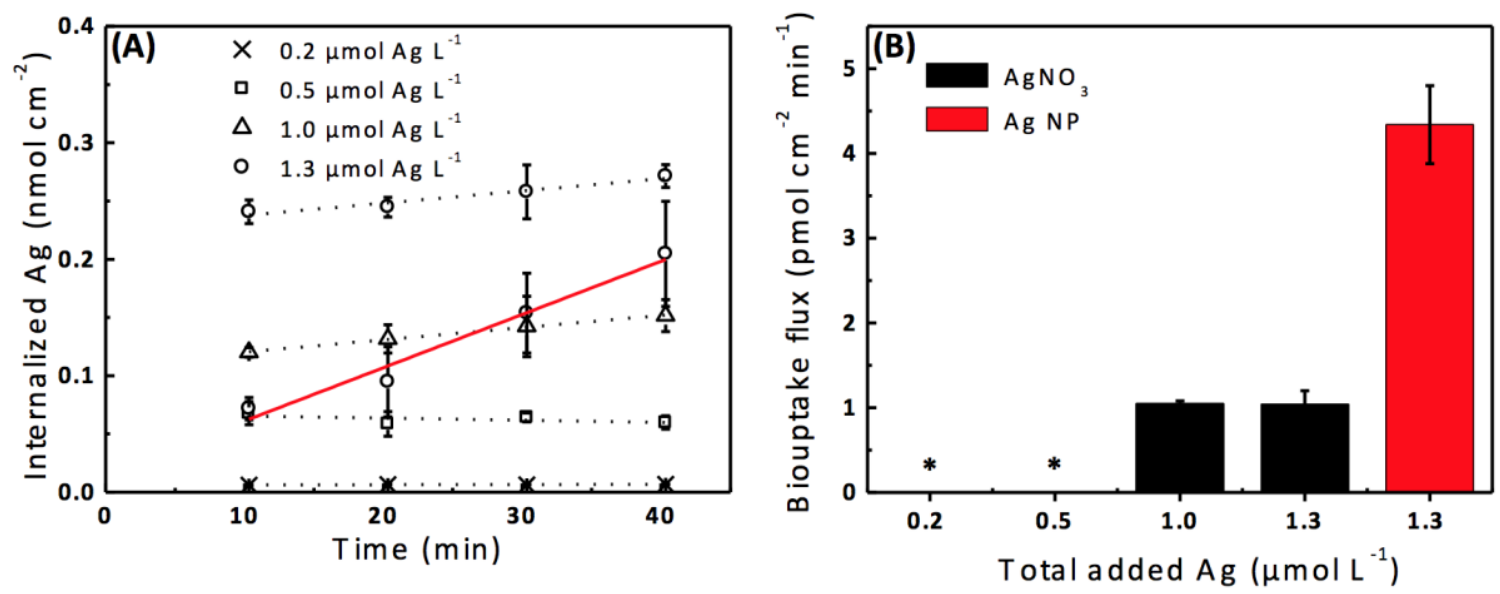

Figure 5. A) Measured Ag biouptake of algal cells (C. reinhardtii) (mean \pm standard deviation, $\mathrm{n}=3$ ) upon exposure to $\mathrm{AgNO}_{3}$ of $0.2,0.5,1.0$ and $1.3 \mu \mathrm{mol} \mathrm{L}-1$, and $\mathrm{Ag} \mathrm{NPs}$ of $1.3 \mu \mathrm{mol} \mathrm{\textrm {L } ^ { - 1 }}$ in wastewater effluents and $\mathrm{B}$ ) corresponding biouptake fluxes (mean \pm standard error, $n=3$ ) for each exposure. Solid (red) biouptake curve refers to Ag NP, while dotted curves refer to $\mathrm{AgNO}_{3}$. Different symbols represent different $\mathrm{Ag}$ concentrations $\left(\mathrm{AgNO}_{3}\right.$ or $\left.\mathrm{Ag} \mathrm{NP}\right)$. Biouptake fluxes (B) correspond to the slopes determined from the internalization curves (i.e. Fig. 5A). (*) denotes fluxes determined for exposures to $\mathrm{AgNO}_{3}$ that were not significantly different $(\mathrm{p}<0.05)$ from zero.

Table 1. Dissolution of Ag NPs in a wastewater effluent medium after 1 day of exposure as characterized by ultracentrifugation experiments $(280,000 g ; 15$ mins). Dissolved Ag is expressed as percentage of the total Ag determined before ultracentrifugation.

\begin{tabular}{cccc}
$\begin{array}{c}\text { Total Ag } \\
\mu g L^{-1}\end{array}$ & \multicolumn{3}{c}{$\begin{array}{c}\text { Dissolution } \\
\%\end{array}$} \\
\hline 25 & 1.4 & \pm & 0.4 \\
50 & 1.9 & \pm & 0.2 \\
200 & 1.9 & \pm & 0.1 \\
\hline
\end{tabular}




\section{References}

1. Chernousova, S. and M. Epple, Silver as Antibacterial Agent: Ion, Nanoparticle, and Metal. Angewandte Chemie International Edition, 2013. 52(6): p. 1636-1653.

2. Lansdown, A.B., Silver in health care: antimicrobial effects and safety in use. Curr Probl Dermatol, 2006. 33: p. 17-34.

3. Morones, J.R., et al., The bactericidal effect of silver nanoparticles. Nanotechnology, 2005. 16(10): p. 2346.

4. Vance, M.E., et al., Nanotechnology in the real world: Redeveloping the nanomaterial consumer products inventory. Beilstein Journal of Nanotechnology, 2015. 6: p. 17691780.

5. Brar, S.K., et al., Engineered nanoparticles in wastewater and wastewater sludgeEvidence and impacts. Waste management, 2010. 30(3): p. 504-520.

6. Gottschalk, F., et al., Modeling flows and concentrations of nine engineered nanomaterials in the Danish environment. International journal of environmental research and public health, 2015. 12(5): p. 5581-5602.

7. Gottschalk, F., T. Sun, and B. Nowack, Environmental concentrations of engineered nanomaterials: review of modeling and analytical studies. Environ Pollut, 2013. 181: p. 287-300.

8. Hendren, C.O., et al., Modeling nanomaterial fate in wastewater treatment: Monte Carlo simulation of silver nanoparticles (nano-Ag). Science of The Total Environment, 2013. 449: p. 418-425.

9. Kaegi, R., et al., Fate and transformation of silver nanoparticles in urban wastewater systems. Water research, 2013. 47(12): p. 3866-3877.

10. Klaine, S.J., et al., Nanomaterials in the environment: behavior, fate, bioavailability, and effects. Environmental Toxicology and Chemistry, 2008. 27(9): p. 1825-1851.

11. Johnson, A.C., et al., Particulate and colloidal silver in sewage effluent and sludge discharged from British wastewater treatment plants. Chemosphere, 2014. 112: p. 4955.

12. Colvin, V.L., The potential environmental impact of engineered nanomaterials. Nature biotechnology, 2003. 21(10): p. 1166-1170.

13. Lowry, G.V., et al., Transformations of Nanomaterials in the Environment. Environmental Science \& Technology, 2012. 46(13): p. 6893-6899.

14. Wiesner, M.R., et al., Decreasing Uncertainties in assessing environmental exposure, risk, and ecological implications of nanomaterialstł. Environmental Science \& Technology, 2009. 43(17): p. 6458-6462.

15. Navarro, E., et al., Environmental behavior and ecotoxicity of engineered nanoparticles to algae, plants, and fungi. Ecotoxicology, 2008. 17(5): p. 372-386.

16. Behra, R., et al., Bioavailability of silver nanoparticles and ions: from a chemical and biochemical perspective. Journal of The Royal Society Interface, 2013. 10(87): p. 20130396.

17. Fabrega, J., et al., Silver nanoparticle impact on bacterial growth: effect of $p H$, concentration, and organic matter. Environmental science \& technology, 2009. 43(19): p. 7285-7290.

18. Miao, A.-J., et al., Intracellular uptake: a possible mechanism for silver engineered nanoparticle toxicity to a freshwater alga Ochromonas danica. PLoS One, 2010. 5(12): p. e15196.

19. Navarro, E., et al., Toxicity of silver nanoparticles to Chlamydomonas reinhardtii. Environmental science \& technology, 2008. 42(23): p. 8959-8964. 
20. Piccapietra, F., et al., Intracellular silver accumulation in Chlamydomonas reinhardtii upon exposure to carbonate coated silver nanoparticles and silver nitrate. Environmental science \& technology, 2012. 46(13): p. 7390-7397.

21. Li, X., et al., Silver nanoparticle toxicity and association with the alga Euglena gracilis. Environmental Science: Nano, 2015. 2(6): p. 594-602.

22. Leclerc, S. and K.J. Wilkinson, Bioaccumulation of Nanosilver by Chlamydomonas reinhardtii - Nanoparticle or the Free Ion? Environmental science \& technology, 2013. 48(1): p. 358-364.

23. Levard, C., et al., Environmental transformations of silver nanoparticles: impact on stability and toxicity. Environmental science \& technology, 2012. 46(13): p. 69006914.

24. Azodi, M., Y. Sultan, and S. Ghoshal, Dissolution Behavior of Silver Nanoparticles and Formation of Secondary Silver Nanoparticles in Municipal Wastewater by SingleParticle ICP-MS. Environmental Science \& Technology, 2016. 50(24): p. 13318-13327.

25. Merdzan, V., et al., The effects of different coatings on zinc oxide nanoparticles and their influence on dissolution and bioaccumulation by the green alga, C. reinhardtii. Science of The Total Environment, 2014. 488-489: p. 316-324.

26. Wilkinson, K.J. and J. Buffle, Critical Evaluation of Physicochemical Parameters and Processes for Modelling the Biological Uptake of Trace Metals in Environmental (Aquatic) Systems, in Physicochemical Kinetics and Transport at Biointerfaces. 2004, John Wiley \& Sons, Ltd. p. 445-533.

27. Yue, Y., et al., Silver nanoparticle-protein interactions in intact rainbow trout gill cells. Environmental Science: Nano, 2016.

28. Impellitteri, C.A., T.M. Tolaymat, and K.G. Scheckel, The speciation of silver nanoparticles in antimicrobial fabric before and after exposure to a hypochlorite/detergent solution. Journal of environmental quality, 2009. 38(4): p. 1528-1530.

29. Kent, R.D., J.G. Oser, and P.J. Vikesland, Controlled evaluation of silver nanoparticle sulfidation in a full-scale wastewater treatment plant. Environmental science \& technology, 2014. 48(15): p. 8564-8572.

30. Liu, J., et al., Controlled Release of Biologically Active Silver from Nanosilver Surfaces. ACS Nano, 2010. 4(11): p. 6903-6913.

31. Sigg, L., et al., Chemical aspects of nanoparticle ecotoxicology. CHIMIA International Journal for Chemistry, 2014. 68(11): p. 806-811.

32. Odzak, N., et al., Dissolution of metal and metal oxide nanoparticles in aqueous media. Environmental Pollution, 2014. 191: p. 132-138.

33. Yue, Y., et al., Toxicity of silver nanoparticles to a fish gill cell line: Role of medium composition. Nanotoxicology, 2015. 9(1): p. 54-63.

34. Sigg, L. and U. Lindauer, Silver nanoparticle dissolution in the presence of ligands and of hydrogen peroxide. Environmental Pollution, 2015. 206: p. 582-587.

35. Groh, K.J., et al., Critical influence of chloride ions on silver ion-mediated acute toxicity of silver nanoparticles to zebrafish embryos. Nanotoxicology, 2015. 9(1): p. 81-91.

36. Yue, Y., et al., Silver nanoparticles inhibit fish gill cell proliferation in protein-free culture medium. Nanotoxicology, 2016. 10(8): p. 1075-1083.

37. Collin, B., et al., Effect of natural organic matter on dissolution and toxicity of sulfidized silver nanoparticles to Caenorhabditis elegans. Environmental Science: Nano, 2016. 3(4): p. 728-736.

38. $\mathrm{Wu}, \mathrm{F} ., \mathrm{B} . J$. Harper, and S.L. Harper, Differential dissolution and toxicity of surface functionalized silver nanoparticles in small-scale microcosms: impacts of community complexity. Environmental Science: Nano, 2017. 
39. McIntyre, A. and C. Guéguen, Binding interactions of algal-derived dissolved organic matter with metal ions. Chemosphere, 2013. 90(2): p. 620-626.

40. Chambers, B.A., et al., Effects of chloride and ionic strength on physical morphology, dissolution, and bacterial toxicity of silver nanoparticles. Environmental science \& technology, 2013. 48(1): p. 761-769.

41. Gondikas, A.P., et al., Cysteine-induced modifications of zero-valent silver nanomaterials: implications for particle surface chemistry, aggregation, dissolution, and silver speciation. Environmental science \& technology, 2012. 46(13): p. 70377045.

42. Zhao, C.-M. and K.J. Wilkinson, Biotic Ligand Model Does Not Predict the Bioavailability of Rare Earth Elements in the Presence of Organic Ligands. Environmental Science \& Technology, 2015. 49(4): p. 2207-2214.

43. Campbell, P.G.C., et al., Metal bioavailability to phytoplankton-applicability of the biotic ligand model. Comparative Biochemistry and Physiology Part C: Toxicology \& Pharmacology, 2002. 133(1): p. 189-206.

44. Fortin, C. and P.G.C. Campbell, Silver uptake by the green alga Chlamydomonas reinhardtii in relation to chemical speciation: Influence of chloride. Environmental Toxicology and Chemistry, 2000. 19(11): p. 2769-2778.

45. Auffan, M., et al., Salinity-dependent silver nanoparticle uptake and transformation by Atlantic killifish (Fundulus heteroclitus) embryos. Nanotoxicology, 2014. 8(sup1): p. 167-176.

46. Khan, Z. and A. Talib, Growth of different morphologies (quantum dots to nanorod) of Ag-nanoparticles: role of cysteine concentrations. Colloids and Surfaces B: Biointerfaces, 2010. 76(1): p. 164-169.

47. Akaighe, N., et al., Humic acid-induced silver nanoparticle formation under environmentally relevant conditions. Environmental science \& technology, 2011. 45(9): p. 3895-3901.

48. Kola, H. and K.J. Wilkinson, Cadmium uptake by a green alga can be predicted by equilibrium modelling. Environmental science \& technology, 2005. 39(9): p. 30403047.

49. Hadioui, M., S. Leclerc, and K.J. Wilkinson, Multimethod quantification of Ag+ release from nanosilver. Talanta, 2013. 105: p. 15-19.

50. Hadioui, M., C. Peyrot, and K.J. Wilkinson, Improvements to single particle ICPMS by the online coupling of ion exchange resins. Analytical chemistry, 2014. 86(10): p. 46684674.

51. Hadioui, M., V. Merdzan, and K.J. Wilkinson, Detection and Characterization of ZnO Nanoparticles in Surface and Waste Waters Using Single Particle ICPMS. Environmental Science \& Technology, 2015. 49(10): p. 6141-6148.

52. Sigg, L. and U. Lindauer, Silver nanoparticle dissolution in the presence of ligands and of hydrogen peroxide. Environ Pollut, 2015. 206: p. 582-7.

53. Hassler, C.S., V.I. Slaveykova, and K.J. Wilkinson, Discriminating between intra- and extracellular metals using chemical extractions. Limnology and Oceanography: Methods, 2004. 2(7): p. 237-247.

54. Pillai, S., et al., Linking toxicity and adaptive responses across the transcriptome, proteome, and phenotype of Chlamydomonas reinhardtii exposed to silver. Proceedings of the National Academy of Sciences, 2014. 111(9): p. 3490-3495.

55. Andersson, L.O., Study of some silver-thiol complexes and polymers: Stoichiometry and optical effects. Journal of Polymer Science Part A-1: Polymer Chemistry, 1972. 10(7): p. 1963-1973. 
56. Laborda, F., et al., Critical considerations for the determination of nanoparticle number concentrations, size and number size distributions by single particle ICP-MS. Journal of Analytical Atomic Spectrometry, 2013. 28(8): p. 1220-1232.

57. Liu, J. and R.H. Hurt, Ion release kinetics and particle persistence in aqueous nano-silver colloids. Environmental science \& technology, 2010. 44(6): p. 2169-2175.

58. Fréchette-Viens, L., M. Hadioui, and K.J. Wilkinson, Practical limitations of single particle ICP-MS in the determination of nanoparticle size distributions and dissolution: case of rare earth oxides. Talanta, 2017. 163: p. 121-126.

59. Adams, N.W.H. and J.R. Kramer, Silver speciation in wastewater effluent, surface waters, and pore waters. Environmental Toxicology and Chemistry, 1999. 18(12): p. 26672673.

60. Martinolich, A.J., et al., Structural and functional effects of Cu metalloprotein-driven silver nanoparticle dissolution. Environmental science \& technology, 2012. 46(11): p. 6355-6362.

61. Auffan, M., et al., Towards a definition of inorganic nanoparticles from an environmental, health and safety perspective. Nature nanotechnology, 2009. 4(10): p. 634-641.

62. Fan, C., et al., Relationship between solubility and solubility product: The roles of crystal sizes and crystallographic directions. Geochimica et cosmochimica acta, 2006. 70(15): p. 3820-3829.

63. Imai, A., et al., Characterization of dissolved organic matter in effluents from wastewater treatment plants. Water Research, 2002. 36(4): p. 859-870.

64. Ma, H., H.E. Allen, and Y. Yin, Characterization of isolated fractions of dissolved organic matter from natural waters and a wastewater effluent. Water research, 2001. 35(4): p. 985-996.

65. Massé, D. and L. Masse, Characterization of wastewater. Canadian Agricultural Engineering, 2000. 42(3).

66. He, D., J.J. Dorantes-Aranda, and T.D. Waite, Silver nanoparticle • algae interactions: oxidative dissolution, reactive oxygen species generation and synergistic toxic effects. Environmental science \& technology, 2012. 46(16): p. 8731-8738.

67. Kroll, A., et al., Extracellular polymeric substances (EPS) of freshwater biofilms stabilize and modify CeO 2 and $\mathrm{Ag}$ nanoparticles. PLoS One, 2014. 9(10): p. e110709.

68. Dupont, C.L. and B.A. Ahner, Effects of copper, cadmium, and zinc on the production and exudation of thiols by Emiliania huxleyi. Limnology and oceanography, 2005. 50(2): p. 508-515.

69. Zhao, C.-M., P.G.C. Campbell, and K.J. Wilkinson, When are metal complexes bioavailable? Environmental Chemistry, 2016. 13(3): p. 425-433.

70. Reinfelder, J.R. and S.I. Chang, Speciation and Microalgal Bioavailability of Inorganic Silver. Environmental Science \& Technology, 1999. 33(11): p. 1860-1863.

71. Yang, G., et al., The role of complexation and competition in the biouptake of europium by a unicellular alga. Environmental toxicology and chemistry, 2014. 33(11): p. 26092615.

72. Chen, Z., L. Zhu, and K.J. Wilkinson, Validation of the biotic ligand model in metal mixtures: Bioaccumulation of lead and copper. Environmental science \& technology, 2010. 44(9): p. 3580-3586.

73. Fortin, C., F.H. Denison, and J. Garnier-Laplace, Metal-phytoplankton interactions: Modeling the effect of competing ions $(\mathrm{H}+, \mathrm{Ca} 2+$, and $\mathrm{Mg} 2+)$ on uranium uptake. Environmental Toxicology and Chemistry, 2007. 26(2): p. 242-248. 